\title{
Void Elimination in Screen Printed Thick Film Dielectric Pastes
}

\author{
S. WILson ${ }^{1}$, S.D. Howison ${ }^{2} \dagger$ and D.F. PARKER ${ }^{3}$ \\ ${ }^{1}$ University of Strathclyde \\ ${ }^{2}$ University of Oxford, UK \\ ${ }^{3}$ University of Edinburgh
}

(Communicated to MIIR on 2 October 2021)

Study Group: ESGI 29, Mar 18-22, 1996, Oxford, UK

Communicated by: C. Breward

Industrial Partner: Du Pont Electronics

Team Members: S. Wilson, University of Strathclyde; S. Howison, University of Oxford; D. Parker, University of Edinburgh; A. Fitt, University of Southampton; O. Harlen, University of Leeds; T. Myers, University of Oxford; C. Please, University of Southampton; E. J. Hinch, University of Cambridge; S.J. Chapman, University of Oxford; J. King, University of Nottingham; G. Duursma, University of Oxford; F. van Beckum, University of Twente; D. Riley, University of Nottingham.

Industrial Sector: Electronics

Key Words: Screen printing, Defects, Surface tension, Lubrication, Tanner's law, Cavitation

MSC2020 Codes: 74, 76

\section{Summary}

The wet screen printing process can be modelled by paste being pushed through a mesh onto a substrate and conductors below. During the process defects, such as vias, voids, pinholes, craters and bubbles can form and evolve. The primary objective of the Study group was to know how best to alter the properties of the paste (rather than the geometry of the screen printing process itself) in order to eliminate the defects. With these goals in mind this report describes a simple model for the closure of craters, a model for the partial closure of vias, a possible mechanism for the formation of pinholes and finally a more detailed consideration of the screen printing process.

$\dagger$ Corresponding Author: howison@maths.ox.ac.uk 


\title{
Void elimination in screen printed thick film dielectric pastes
}

\author{
Du Pont Electronics
}

\section{The Industrial Process}

Figure 1 summarises the geometry of the wet screen printing process. A semi-rigid rubber squeegee pushes the paste through the woven wire mesh and onto the substrate and conductors below. Typical dimensions are indicated on the Figure. The pastes used are concentrated dispersions of particles with mean diameter $3 \mu \mathrm{m}$ and maximum diameter $15 \mu \mathrm{m}$, and are rheologically complicated. Typically they are shear-thinning, display a critical yield stress (below which they do not flow) of $\sigma_{c} \simeq 300 \mathrm{~Pa}$, have density $\rho \simeq 10^{3}$ $\mathrm{kg} / \mathrm{m}^{3}$ and surface tension $\gamma \simeq 40 \mathrm{mN} / \mathrm{m}$. Holes in the applied paste layer can be classified as follows.

- A via is a large two-dimensional channel created intentionally by "blanking out" areas of the mesh. These are observed to close up partially after printing, which is undesirable unless it can be controlled precisely.

- A void is a large two-dimensional hole created accidentally near the leading edge of a conductor. These can usually be eliminated by adjusting the printing process and so were not considered further.

- A pinhole is a small approximately circular hole in the layer of radius $R \simeq 20 \mu \mathrm{m}$ which extends right through to the substrate. Pinholes can persist as the layer dries and are the main cause of concern to DuPont.

- A crater similar to a pinhole except that it does not extend right through the layer to the substrate. Typically craters close up as the layer dries.

- A bubble. Typically these are caused by accidental air entrapment during the printing process. Bubbles are not a major concern to DuPont and so were not considered further.

Many small holes are inevitably formed in the paste layer as it is squeezed through the mesh, but most (approximately $90 \%$ ) quickly close. An important physical observation is that those holes which survive as pinholes originate, as shown in Figure 1, about two mesh diameters (typically $L=100 \mu \mathrm{m}$ ) upstream of the leading edge of the conductor.

Using the typical values quoted above the non-dimensional Bond number $\rho g L^{2} / \gamma=$ $2.5 \times 10^{-3}$ (where $L=100 \mu \mathrm{m}$ is the typical horizontal lengthscale of the flow) is small and so gravity effects may be neglected. This agrees with practical experience as the orientation of the coated components during drying makes no significant difference to the final product. 


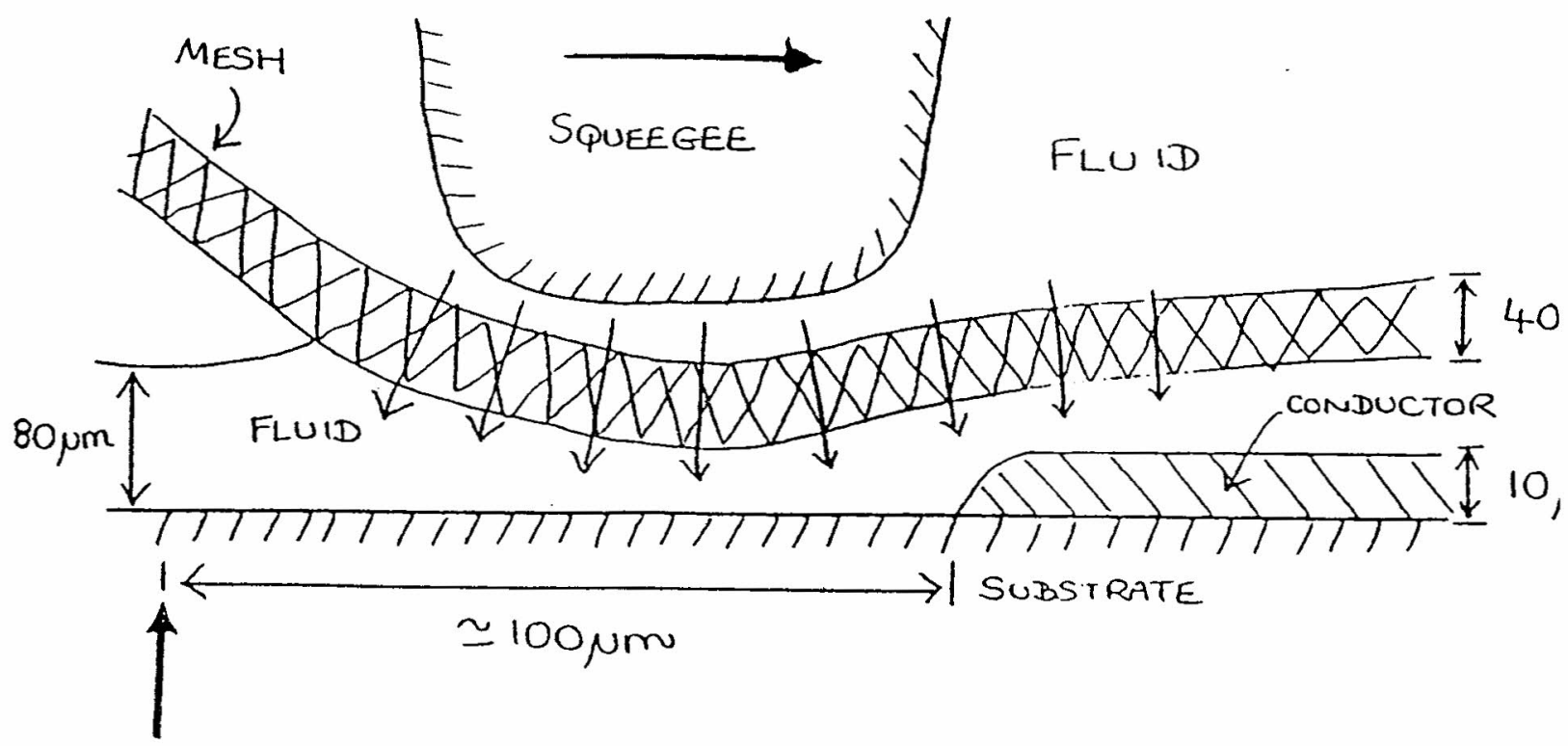

Pinholes

FORM HERE

Figure 1: Geometry of the screen printing process (not to scale!)

\section{The Problem}

The problem is to understand the mechanisms for the formation and evolution of defects in wet screen printed layers. The primary objective is to know how best to alter the properties of the paste (rather than the geometry of the screen printing process itself) in order to eliminate the defects. With these goals in mind the work done during the Study Group reported here was as follows; to describe a simple model for the closure of craters, a model for the partial closure of vias, a possible mechanism for the formation of pinholes and finally a more detailed consideration of the screen printing process.

\section{A model for the closure of craters}

A typical crater is shown in Figure 2(a). The closure of craters can easily be explained in terms of mean surface tension effects. At the bottom of the crater both principal curvatures are of the same sign and hence surface tension acts to close it. However, they may be held open as a result of the presence of the critical yield stress. For the idealised hemispherical crater with radius $R$ shown in Figure 2(b) the force due to mean surface tension will be comparable to the yield stress if

$$
\frac{2 \gamma}{R} \simeq \sigma_{c}
$$

which would imply that $R \simeq 50 \mu \mathrm{m}$. However typically $R=10-25 \mu \mathrm{m}$ and so, in agreement with observation, all craters are expected to close. 

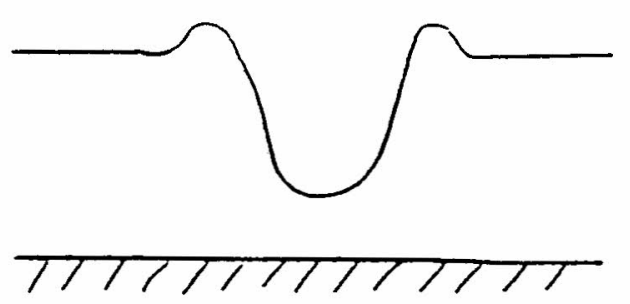

(a)

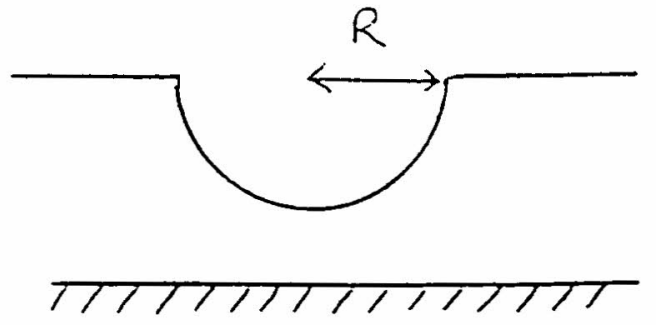

(b)

Figure 2: (a) A typical crater (b) An idealised crater

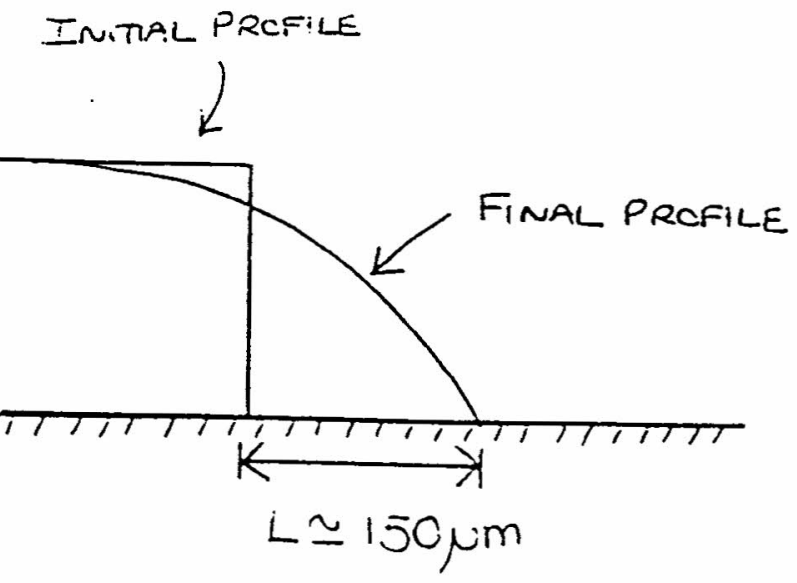

Figure 3: The edge of a via 


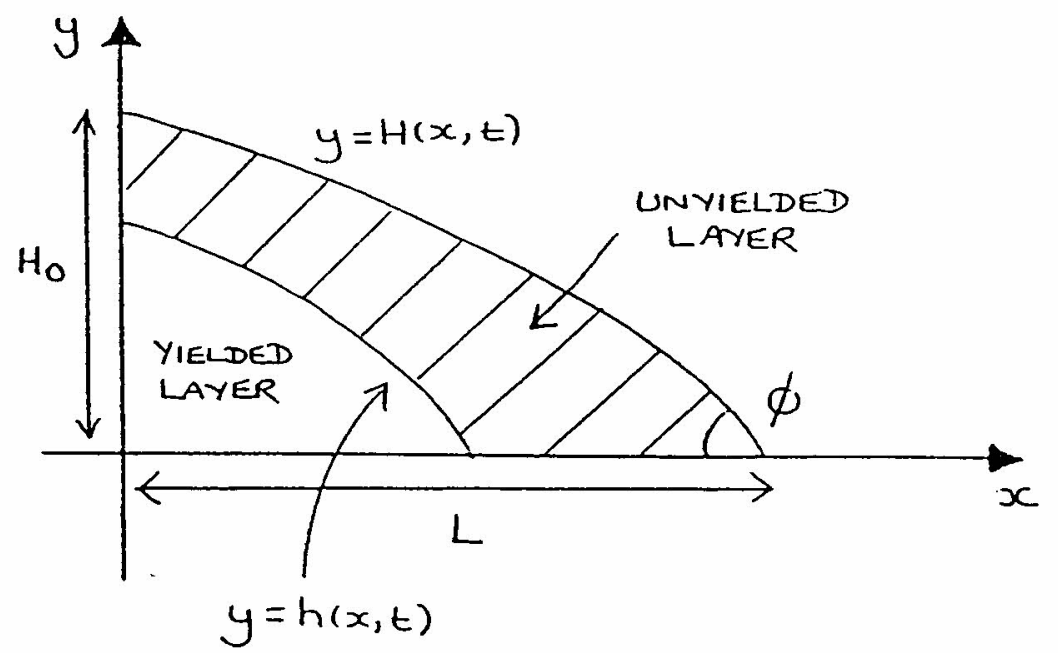

Figure 4: Mathematical model for the motion of the edge of a via

\section{A model for the partial closure of vias}

As shown in Figure 3, the edges of vias are observed to flow inwards after printing by $L \simeq 150 \mu \mathrm{m}$. We suggest that this behaviour is essentially a wetting problem, with the motion of the contact line driven by the variation of the dynamic contact angle. However, the motion usually stops before the static contact angle is reached, indicating that yield stress also plays a role.

We propose a simple mathematical model for the motion of the edge of a via based on classical lubrication theory (and hence appropriate if the profile is sufficiently slender). As shown in Figure 4 the flow consists of a yielded region $\left(\sigma>\sigma_{c}\right)$ near the substrate with upper boundary $y=h(x, t)$ and an unyielded region $\left(\sigma<\sigma_{c}\right)$ near the surface of the paste $y=H(x, t)$. In the yielded region the classical lubrication approximation yields the familiar equations

$$
\dot{p}_{x}=\mu u_{y y}, \quad p_{y}=0, \quad u_{x}+v_{y}=0
$$

for the pressure $p$ and velocity $(u, v)$ and so

$$
u=\frac{p_{x}}{2 \mu}\left(y^{2}-2 h y\right)
$$

satisfying $u=0$ on $y=0$ and $u_{y}=0$ on $y=h$. Hence the flux in the yielded region,

$$
Q_{1}=\int_{0}^{h} u \mathrm{~d} y=-\frac{h^{3} p_{x}}{3 \mu} .
$$

The unyielded layer moves locally as rigid block with speed ${ }^{1}$

$$
u=-\frac{h^{2} p_{x}}{2 \mu}
$$

\footnotetext{
${ }^{1}$ Clearly this speed is not constant! the difficulty arises from a conflict between the "thin-film" limit and the limiting process leading to the Bingham model and could be overcome by a more careful analysis. However, for the purposes of this simple model we simply ignore it!
} 
and so

$$
Q_{2}=\int_{h}^{H} u \mathrm{~d} y=-(H-h) \frac{h^{2} p_{x}}{2 \mu} .
$$

Conservation of mass requires that

$$
H_{t}+Q_{x}=0
$$

where

$$
Q=Q_{1}+Q_{2}=\frac{h^{2} p_{x}}{2 \mu}\left(\frac{h}{3}-H\right),
$$

and the normal stress condition yields $p=-\gamma H_{x x}$. If $h>0$ then the requirement that the stress takes its critical value at the yield line gives

$$
(H-h) p_{x}=\sigma_{c} \quad \text { on } \quad y=h .
$$

However, if $h=0$ then the whole of the layer is unyielded all the way across and the stress on $y=0$ will, in general, be less than the yield stress. Hence the model satisfies the "complementarity" conditions

$$
h \geq 0, \quad(H-h) p_{x}-\sigma_{c} \leq 0, \quad h\left[(H-h) p_{x}-\sigma_{c}\right]=0 .
$$

To close the problem we need to address the flow in the vicinity of the moving contact line $x=L(t)$. In order to avoid a detailed discussion we adopt the semi-empirical Tanner's Law (see L.H. Tanner J. Phys D 12 pp1473-1484 (1979) for example)

$$
\frac{\mathrm{d} L}{\mathrm{~d} t}=K\left(\phi^{3}-\phi_{0}^{3}\right)
$$

where $\phi_{0}$ is the static value of $\phi$ and $K$ is an empirically-determined constant.

Rather than attempt a full numerical treatment of this problem we just consider the possible steady solutions. In equilibrium $Q=0$ and so either $p_{x}=0$ or $h=0$. In the former case the profile is parabolic and independent of $\sigma_{c}$, while in the latter case the layer is unyielded all the way across and the conditions given in equation (9) imply that

$$
H_{x x x} \leq-\frac{\sigma_{c}}{\gamma H} .
$$

If $h=0$ everywhere (in which case the entire layer is unyielded) and if the inequality (11) is an equality then $H$ satisfies the third order differential equation

$$
H_{x x x}=-\frac{\sigma_{c}}{\gamma H} \text {. }
$$

We expect the solution of equation (12) to be an upper bound on the final spread of the via. If $H_{0}$ is the height of the via at $x=0$ then equation (12) means that the appropriate scale for $L$, denoted by $[L]$, is given by $H_{0} /[L]^{3}=\sigma_{c} / \gamma H_{0}$ and so

$$
[L]=\left(\frac{\gamma H_{0}^{2}}{\sigma_{c}}\right)^{\frac{1}{3}} .
$$

For the typical values quoted above $[L]=95 \mu \mathrm{m}$. Scaling $x$ with $[L]$ and $y$ with $H_{0}$ equation (12) becomes (in non-dimensional variables)

$$
H_{x x x}=-\frac{1}{H}
$$




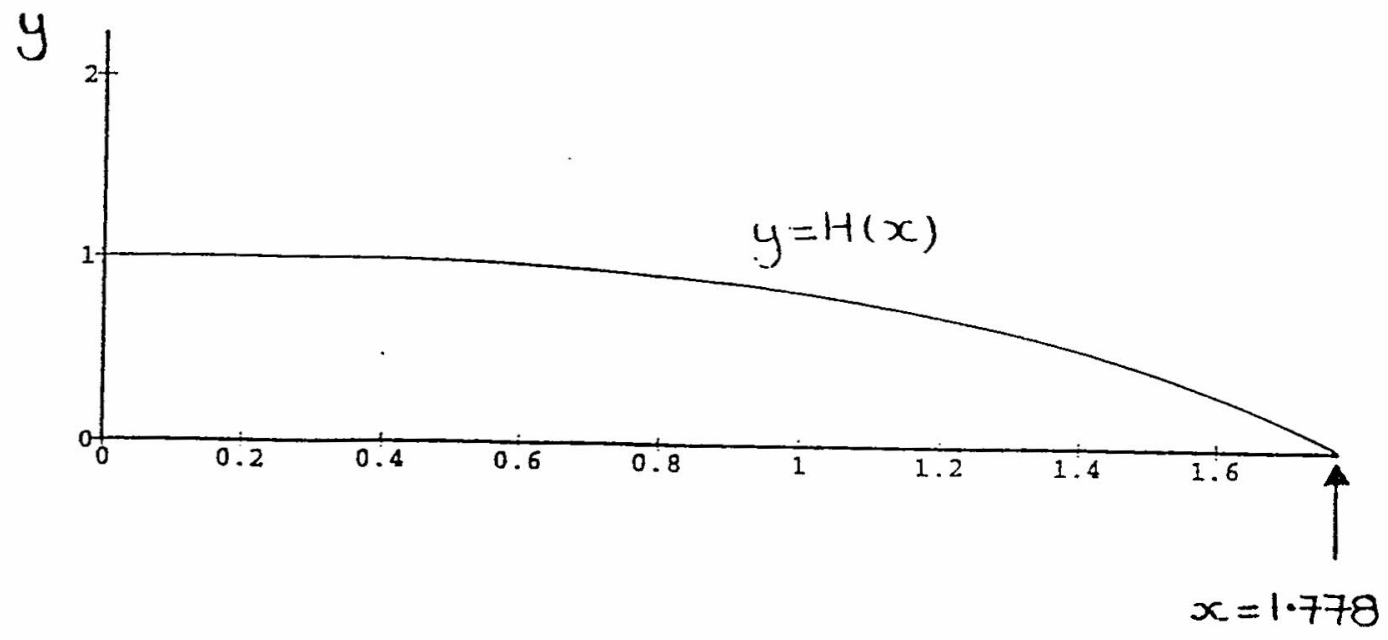

Figure 5: Numerically calculated solution of equation (14)

subject to the boundary conditions $H(0)=1, H_{x}(0)=0$ and $H_{x x}(0)=0$. Figure 5 shows the result of solving equation (14) numerically. ${ }^{2}$ In particular this calculation shows that $H=0$ at $x=1.778$, and so our final prediction for the maximum extent of the spread of a via is

$$
L=1.778\left(\frac{\gamma H_{0}^{2}}{\sigma_{c}}\right)^{\frac{1}{3}}=168 \mu \mathrm{m},
$$

in excellent agreement with the observed value of approximately $150 \mu \mathrm{m}$. The expression for $[L]$ shows that this value is relatively insensitive to changes in the values of $\gamma$ and $\sigma_{c}$ and that reducing $H_{0}$ (the height of the via) may be the only practical way to control the size of the spread. Clearly experiments aimed at verifying equation (15) would be worthwhile.

\section{Pinholes}

\subsection{Basic Considerations}

As Figure 6 shows, unlike craters, the principal curvatures of a pinhole are always of different sign and hence non-trivial equilibrium shapes (described by the classical LaplaceYoung equation) are possible. Taylor \& Michael [J. Fluid Mech. 58, 625-639 (1973)] showed that for all values of the static contact angle $0<\phi \leq \pi$ the thickness of the layer $h$ is monotonically increasing function of hole radius $R$ satisfying $h \rightarrow 0$ as $R \rightarrow 0$ and $h \rightarrow h_{c}=2(\gamma / \rho g)^{1 / 2} \sin (\phi / 2)$ as $R \rightarrow \infty$, and so there is a unique hole with radius $R$ and contact angle $\phi$ in every layer of thickness $h$ less than the critical value $h_{c}$. However, by considering the energy of these equilibrium solutions Taylor \& Michael showed them to be unconditionally unstable. These calculations include the effects of gravity; in the absence of gravity the thickness of the layer grows without bound far from the hole, but the main conclusion of unconditional instability remains unaltered.

\footnotetext{
${ }^{2}$ Equation (14) can be reduced to a first order ordinary differential equation, but since the numerical solution is straight forward this is not pursued here.
} 


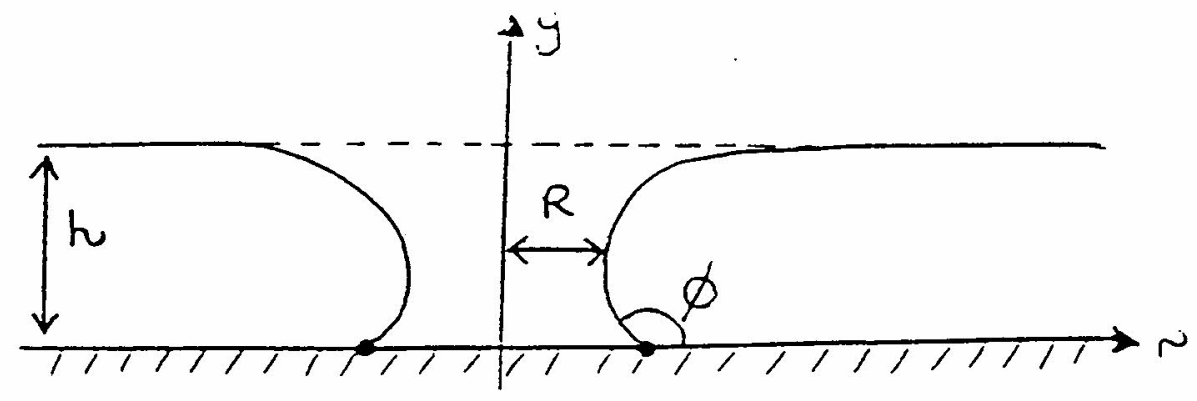

Figure 6: A pinhole

An order of magnitude calculation similar to that for the closure of craters shows that the instability persists in the presence of a yield stress of the relevant magnitude, and so a new mechanism is required to keep a small fraction (approximately $10 \%$ ) of the holes open. We conjecture that the reason pinholes do not close is that the pinhole is surrounded by an annular region in which the volume fraction of solid particles is large enough for them to have locked together to form a rigid ("Roman arch") matrix. (This is certainly feasible since the initial volume fraction can be as high as $25 \%$ and locking occurs at around $35 \%$.) Locking is important because it is considerably more difficult for the resin to flow through the gaps in such a matrix than it is for the particles and resin to move together: this is because in the latter case it is only necessary to shear the fluid in the gaps while in the former resin must be driven through the gaps. It is therefore plausible that a pinhole surrounded by a locked matrix may close so slowly as to remain open until firing takes place.

\subsection{A possible mechanism for the formation of pinholes}

Pinholes form about $300 \mu \mathrm{m}$ upstream of the raised strip of conductor, and are well correlated with the mesh spacing. The purpose of this section is to answer the two questions:

- How do pinholes form?

- Why do they form where they do?

\section{(a) Squeeze film model for formation}

We conjecture that pinholes form at the intersection of two mesh wires, where the mesh is closest to the substrate. The sequence of events as the squeegee passes may be as follows.

1. As the mesh is pressed towards the substrate, the particles suspended in the resin are displaced approximately radially away from the point of closest approach. There are two reasons for this: (i) they are in a pressure gradient, from high at the point of closest approach to low further away; thus there is a pressure drop across each particle and hence an outward force, and (ii) if particles are squeezed close to the wire there is an even higher local pressure which pushes them out (like a squeezed pip).

There is therefore a relatively clear area immediately around the point of closest approach, surrounded by a 'ring' where the density of particles is higher than initially 
(recall from the discussion on the persistence of pinholes that a solid volume fraction of $35 \%$ is enough to lock into a rigid matrix, through which the fluid cannot easily flow).

2. The mesh is lifted. Because slow flow is time-reversible, there must be something to prevent the particles from returning into the clear area. A possible mechanism is cavitation: if the fluid cavitates at about 1 bar below ambient pressure, then the cavity may grow as shown in Figure 7 , to leave a pinhole. Although we have not carried out detailed computations, the minimum pressure is

$$
p_{m} \sim O\left(D \mu \dot{s} / s^{2}\right)
$$

where $D$ is the mesh wire diameter, $s(t)$ the separation between the mesh and the substrate, and $\mu$ is the viscosity. Here

$$
\dot{s} \sim \text { squeegee speed } \times \text { mesh slope }
$$

but is modified by the competition between the tension in the mesh and the pressure (these forces have magnitude of tension $\times$ wire curvature and $D p_{m}$ respectively).

This proposed mechanism predicts that pinholing should be more common if:

- The paste cavitates easily (or a constituent of the solvent vaporises easily);

- The mesh tension is low (so the minimum separation is small and pressures are large);

- The squeegee moves too fast (increasing $\dot{s}$ );

- The paste viscosity is too high.

\section{(b) Pinhole location}

In terms of the paste thickness and especially the step height, the $300 \mu \mathrm{m}$ horizontal offset of pinholes is large, and one would not normally expect to feel the influence of the step as far upstream as this. A possible mechanism that singles out this location is as follows. The idea is that pinholes form where the minimum mesh-substrate separation is smaller than average. As shown in Figure 8 , as the squeegee moves across the step, unusually high pressures are generated in the converging channel at the step edge. The relatively stiff mesh wires act as cantilevers, being pushed up at the step edge where pressures are high, and so down behind the squeegee, a distance upstream that depends on the squeegee's radius of curvature and on the resistance of the mesh wires to bending. It is not yet clear whether the former or the latter controls the squeegee position.

\section{A more detailed consideration of the screen printing pro- cess}

Anecdotal evidence suggests that paste is squeezed through the mesh as jets, concentrated close to the squeegee tip. Can we account for this, as a preliminary to exploring the observation that pinholes occur typically at 300-500 $\mu \mathrm{m}$ from a $12 \mu \mathrm{m}$ step? The dimensions of the mesh (wire diameter, $b=53 \mu \mathrm{m}$; mesh spacing, $a=125 \mu \mathrm{m}$ ) give thickness $=2 b$, cell 


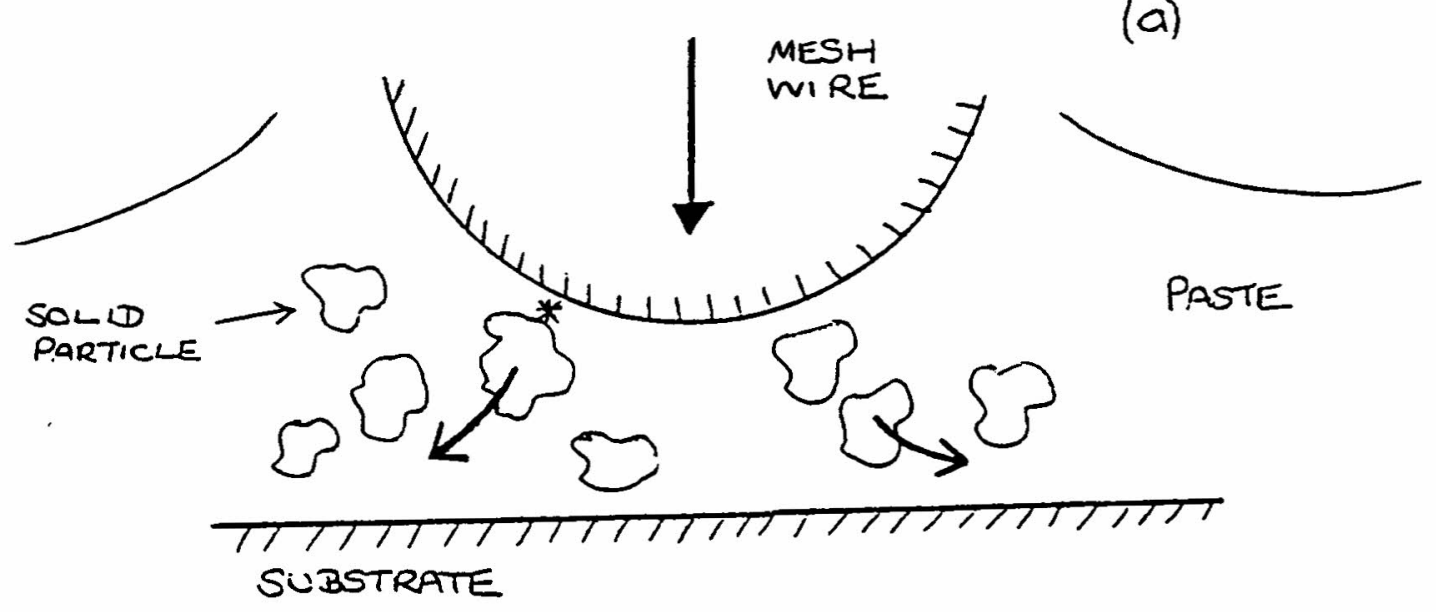

(b)

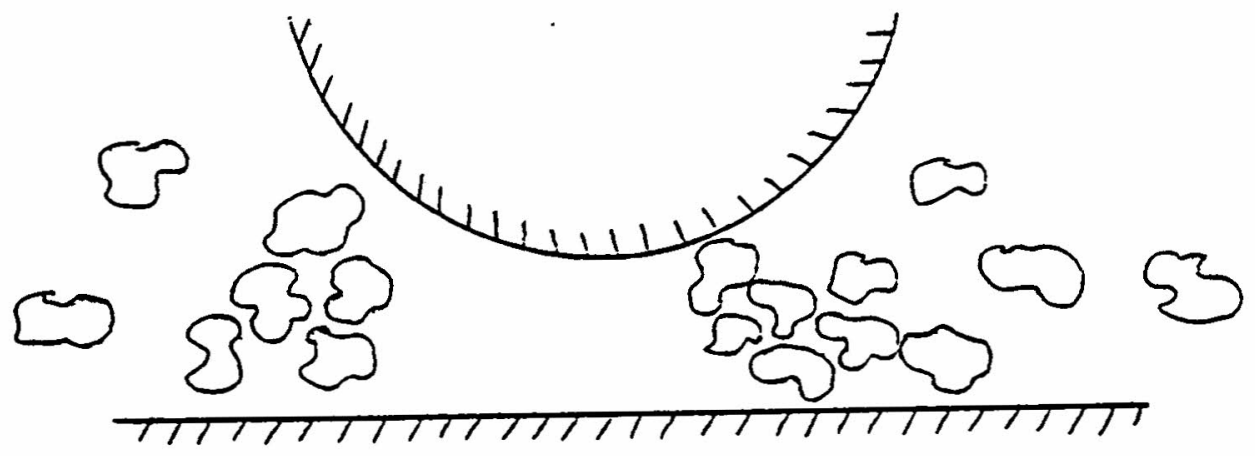

$i$

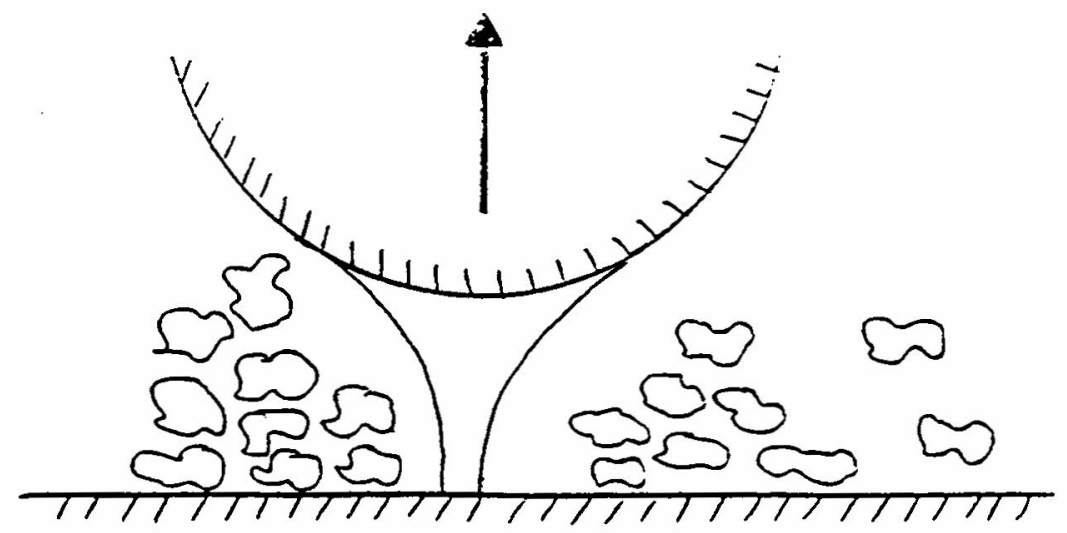

Figure 7: Mechanism for the formation of a cavity: (a) the descending mesh wire pushes particles away, creating high pressure at the point marked with a star (*), (b) at closest approach, (c) a cavity forms as the wire is lifted off 


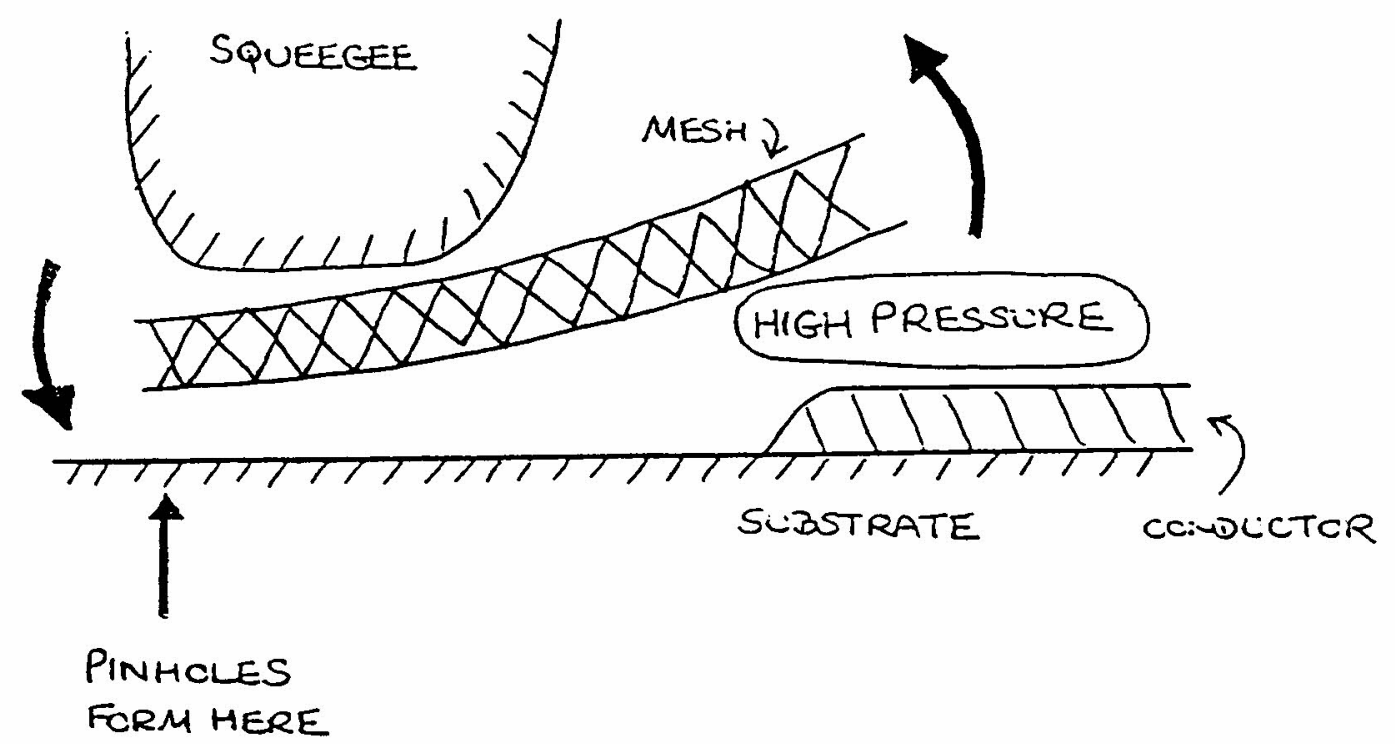

Figure 8: The cantilever mechanism

volume $=2 a^{2} b \equiv V_{c}$, of which the open pore volume $\approx 2 a^{2} b\left\{1-\frac{1}{4} \pi a^{-1} b\left(1+a^{-2} b^{2}\right)^{1 / 2}\right\} \equiv$ $V_{p} \approx 0.64 V_{c}$. However, a wet paste thickness of $\approx 90 \mu \mathrm{m}$ is laid down, while only $\approx 0.64 \times 106=68 \mu \mathrm{m}$ is held within each pore.

Questions that arise include the following. What determines how much of the necessary additional $\approx 22 \mu \mathrm{m}$ of paste thickness passes under the squeegee tip either (a) between the mesh and the substrate, or (b) between the squeegee tip and the mesh? If a significant thickness exists in (b), what controls this thickness? In any case, what determines the rate at which paste is sucked through as the mesh lifts off? The paste extruded ahead of the squeegee as a jet must then be squeezed axially until the jets coalesce to form a film. How does this process control the layer thickness in (a); could this process be influenced by a $12 \mu \mathrm{m}$ step at approximately 3 mesh spacings distant? If so, could gaps (bubbles) provide initiation sites for pinholes during the mesh lift-off?

Figure 9 summarises the paste jet extrusion geometry and a (conjectural) process of paste jet coalescence. Two aspects of this problem and some suggestions for further work are discussed below.

\subsection{Paste jet extrusion}

A jet extrusion model for a shear-thinning fluid, with general relation $\mu=\mu(S)$ between viscosity and maximum shear rate $S$ was developed. This postulates an equivalent axisymmetric pore with radius $R=R(z)$ and relates the value of the shear stress $\sigma$ on the wall, denoted by $\sigma_{w}$, to volume flux $Q$ and $R$ through the equation

$$
\frac{Q}{2 \pi R^{3}}=\frac{M\left(\sigma_{w}\right)}{2 \sigma_{w}^{3}} \quad \text { where } \quad M\left(\sigma_{w}\right)=\int_{0}^{\sigma_{w}} \sigma^{2} F(\sigma) \mathrm{d} \sigma
$$

in which $S=F(\sigma)$ is the inverse of the function $\sigma=S \mu(S)$. Assuming that the model applies for $z_{1}<z<z_{2}$, then the pressure difference across the mesh, $\Delta p$, is given by

$$
\Delta p=\left(\frac{\pi}{Q}\right)^{\frac{1}{3}} \int_{z_{1}}^{z_{2}}\left[M\left(\sigma_{w}\right)\right]^{\frac{1}{3}} \mathrm{~d} z
$$



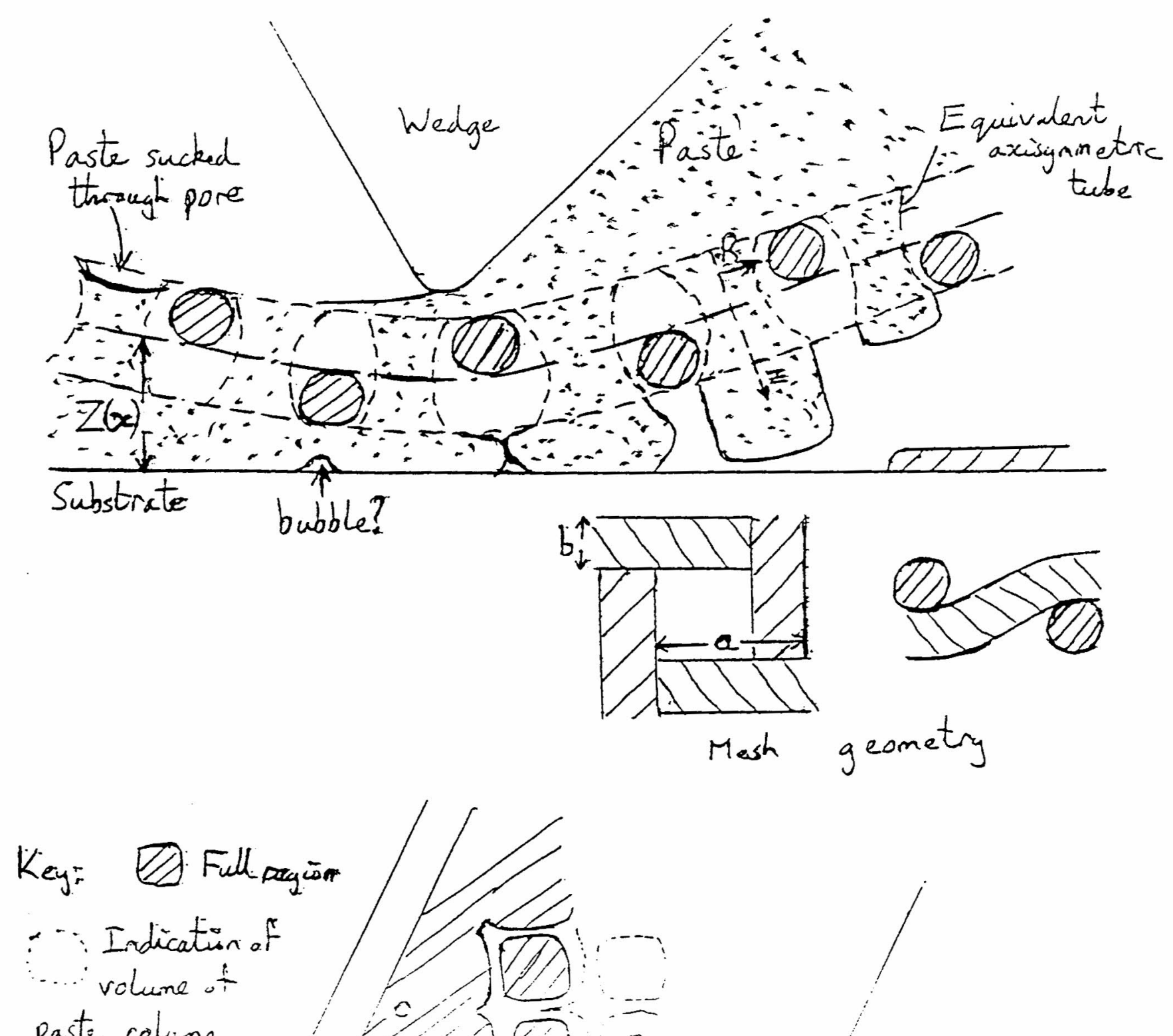

paste colamn

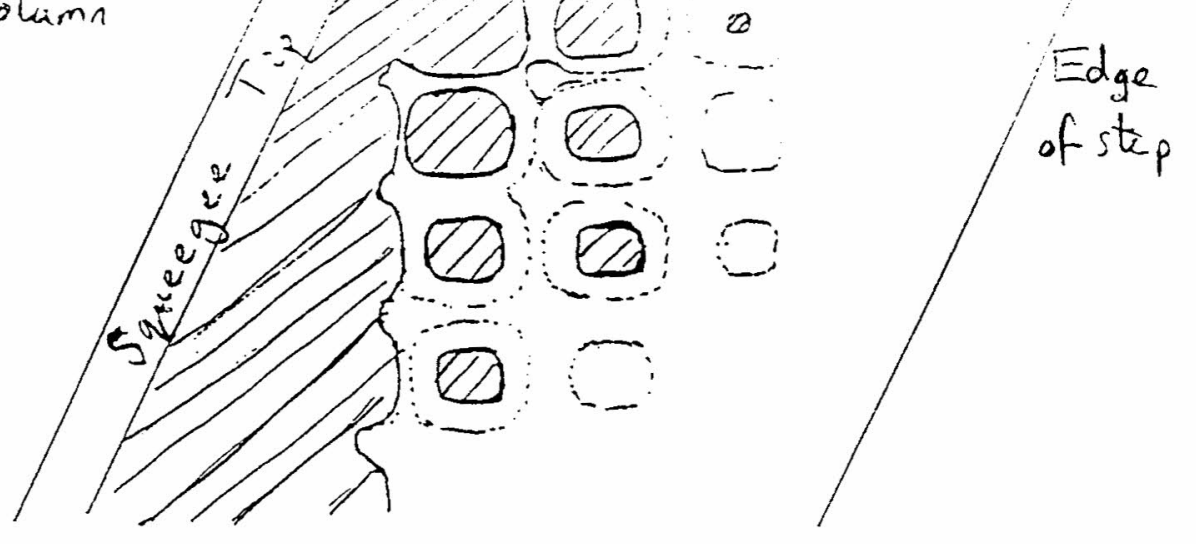

Figure 9: Details of the screen printing process 
For both power law and piecewise linear viscosity laws this model displays a threshold effect in which $Q$ increases rapidly over a relatively small increase in $\Delta p$. Analogous calculations for experimentally valid viscosity laws should confirm that extrusion is concentrated close to the squeegee tip.

\subsection{Paste jet coalescence}

A model for pressure build-up beneath the mesh involving paste jets which are first compressed axially, then coalesce to form a film was also proposed. The jet broadening again exhibits a threshold effect - so predicting considerable 'back-pressure' to inhibit flow through the mesh. Any subsequent high pressure build-up under the squeegee tip would require 3-dimensional computation for a jet constrained by symmetry at the walls of a rectangular cell. This pressure distribution indicates the possibility that bubbles might be trapped, particularly near the substrate surface.

\subsection{Further work}

Possible extensions required are the analysis of paste suction through the mesh as it "lifts off", with $\mid$ slope $\mid \leq 0.01$. Here, below the mesh the paste will be essentially at rest, probably under negative pressure. We can model this by taking $p$ to vary (gradually) with $x$ (and $t$ ). The resulting (small?) pressure difference $\Delta p$ (varying with $x$ and $t$ ) will suck the paste (slowly) through the mesh. As the mesh lifts off, with centreline height $Z=Z(x, t)$, neglect of horizontal flow (not desired) gives

$$
Q=a^{2} \frac{\partial Z}{\partial t}
$$

Also,

$$
Q=-\pi R^{2}\left(z_{1}\right) \frac{d z_{1}}{d t}
$$

where $z=z_{1}(t)$ denotes the upper surface of the pore. Since $\Delta p$ is also related to the mesh geometry by

$$
\Delta p \approx T \frac{\partial^{2} Z}{\partial x^{2}}
$$

a mechanism exists for rapid increase in $Q$ if the mesh develops localised large curvature when the squeegee is forced to rise to mount a $12 \mu \mathrm{m}$ step. Since rapid increase in $Q$ will then suddenly reduce $z_{1}$ locally, this could momentarily induce rapid lift-off. It might be possible to coordinate this mechanism with the idea of pinhole formation by cavity growth from bubbles left at the substrate surface.

Finally, we observe that this description suggests that bubbles exist elsewhere at the paste/substrate interface but without subsequent formation of pinholes. An experimental confirmation of this suggestion (perhaps by deposition onto a glass substrate) would be very interesting!

\section{Acknowledgements}

Many people worked on this problem, including S. Wilson, S. Howison, D. Parker, A. Fitt, O. Harlen, T. Myers, C. Please, E. J. Hinch, J. Chapman, J. King, G. Duursma, F. van Beckum and D. Riley. Report written by SKW, SDH and DFP. 